Mbya Moya disease in 11. An embolism based on congenital heart disease was found in 8 , with trauma in 3 , and with infection in 1 . Cerebral angiography confirmed stenoses or occlusions in 17 of 21 patients tested. The initial symptams of juvenile ischemic cerebral disease was hemiparesis in 22 (47.8\%), convulsion in $9(19.6 \%)$ and speech impairment in $7(15.2 \%)$. The prognosis in patients with an unknown etiology for the occlusion had good outcomes whereas those with congenital heart disease had a relatively poor prognosis. Three patients had abscesses after their ischemic lesions. (Wanifuchi H et al. Ischemic stroke in infancy, childhood and adolescence. Child's Nerv Syst Dec $1988 ; \underline{4}: 361-364$ ).

COMMENT. Cerebral arterial occlusion in children is uncommon and only $3 \frac{\%}{\%}$ of cerebral infarctions occur in patients under the age of 40. A thorough diagnostic search to prevent recurrences is of importance in the young stroke victim. Despite a lengthy differential diagnosis of cerebral infarction several predominant etiologies account for the majority of cases. The category of uncertain etiology includes $35 \%$ of patients in wham the cerebral infarction was associated with mitral valve prolapse, migraine and oral contraceptive use. Each of these conditions is frequent enough in healthy young adults that causality cannot be assumed until other causes have been eliminated (Hart, Miller. Stroke 1983; 14:110). The causes of infarction in 100 young adults were listed as cerebral vascular atherosclerosis (18), cerebral embolism (31), cerebral vasculopathy (10), coagulopathy and systemic inflammation (9), peripartum (5), and uncertain etiology (27). Ergot preparations and oral contraceptives were contraindicated in patients with migraine headaches and cerebral infarction. Platelet antiaggregates were advised in migraine patients with cerebral infarction and in the idiopathic cases. In the present paper fram Tokyo, same of the patients with arterial occlusion of unknown etiology had had frequent episodes of inflammation such as measles or tonsillitis in their past history and the occlusion and inflammation were thought to be indicative of arteritis. Patients with occlusion of the internal carotid artery often show pathological findings consistent with the early stages of Mbya Moya disease in childhood (Suzuki J Takaku A. Arch Neurol 1969; 20:288).

\title{
HYROCEPHALUS
}

\section{BAERS IN CONGENITAL HYDROCEPHALUS}

Brainstem auditory evoked responses were studied in 20 children with congenital hydrocephalus before and after shunt surgery at the Departments of Neurosurgery and Neurology, National Institute of Mental Health and Neurosciences, Bangalore, India. Ninety-five percent showed abnormal responses preoperatively. Prolonged wave V latency was the most common abnormality, followed by increased interwave latencies. Absence of evoked responses was more cammon in children with communicating hydrocephalus. Following shunt surgery $50 \%$ of the responses returned to normal and $20 \%$ showed a significant improvement. The ages of the patients ranged from 2-30 months (mean 8.5). The duration of neurological symptams ranged from 1-12 months (mean 3.9). A progressive increase in head size was the chief sign on presentation. Twelve of the children had congenital hydrocephalus alone and 
8 had hydrocephalus and an associated meninganyelocele. BAER abnormalities were similar in the two groups. Postoperative BAER changes correlated well with reduction in ventricular size as determined by CT. The worsening of BAER postoperatively in 4 patients $(20 \%)$ correlated with an abnormality in the postoperative CT scan, which showed progression of hydrocephalus due to shunt block and slipped shunt tube and subdural hematoma (2). BAER abnormalities referable to dysfunction of the rostral brainstem recovered later than those localized to the caudal brainstem. The proximity of the upper brainstem to the enlarged ventricular system could be responsible for pathological changes such as compression, distortion or displacement leading to associated persistent edema and more prolonged BAER abnormalities. (Venkataramana N K et al. Evaluation of brainstem auditory evoked responses in congenital hydrocephalus. Child's Nerv Syst Dec 1988; 4 :334-338).

COMMENT. The study of BAER is useful for identifying physiological brainstem abnormalities in hydrocephalic children and promises to be a sensitive, noninvasive, diagnostic tool for the detection of complications of shunt surgery other than those secondary to infection. Causes of dysfunction in the brainstem associated with congenital hydrocephalus include distortion and displacement, raised intracranial pressure, and developmental anomalies of the brainstem and auditory pathways. In $20 \%$ of the cases in this study, worsening of BAER postoperatively was correlated with complications such as subdural hematoma and raised intracranial pressure.

BAER IN POSTHEMDRRHAGIC VENTRICULAR DILATATION

Nineteen infants with posthemorrhagic ventricular dilatation were studied with serial auditory brainstem responses at the Department of Paediatrics and Neonatal Medicine, Hammersmith Hospital, London. The cerebrospinal fluid pressure was measured in 9 of the 19 infants directly during lumbar or ventricular taps with a Gaeltic pressure transducer. No correlation was found between cerebrospinal fluid pressure and prolonged interpeak intervals on the BAER. Improvement occurred in 3 patients when cerebrospinal fluid was withdrawn. In one full term infant, improvement in BAER occurred one week after shunting. The lack of correlation between the $I-V$ interpeak interval and the intracranial pressure in preterm infants was probably due to better adaptation of the immature brain to increased intracranial pressure. (Lary S, Dubowitz V et al. Arch Dis Child Jan 1989; 64: 17-23).

COMMENT. Abnormalities in auditory brainstem responses in premature infants may resolve irrespective of the persistence of progression of ventricular dilatation. Improvements in BAER, especially of the amplitude, could occur after drainage of cerebrospinal fluid in same cases. The responses in full term infants may differ from those of preterm infants. 